\title{
Erforderlicher technischer Durchsatz von Materialfluss- systemen zur Erreichung eines Solldurchsatzes - ermittelt durch Simulation mit Iterationsalgorithmus
}

\author{
DIPL.-ING. DR.TECHN. KLAUS DECKER \\ UNIV.-PROF DIPL.-ING. DR.TECHN. BRUNO GRÖSEL \\ INSTITUT FÜR KONSTRUKTIONSWISSENSCHAFTEN UND TECHNISCHE LOGISTIK, WIEN
}

Dieser Artikel beschäftigt sich mit komplexen Materialflusssystemen und Reihenschaltungen von Förder- und Bedienelementen. Diese können durch eine bestimmte Verfügbarkeit charakterisiert werden. Die daraus resultierende Gesamtverfügbarkeit bestimmt den zur Erzielung eines vorgegebenen Durchsatzes erforderlichen „technischen Durchsatz“ der Einzelelemente. Unterliegen die Förder- bzw. Bedienzeiten einer stochastischen Verteilung, so ist die Zwischenschaltung von Puffern erforderlich, die aber auch zur Reduzierung des erforderlichen technischen Durchsatzes aufgrund von Störungen beitragen können. Das Systemverhalten komplexer Anlagen kann nur durch Simulation untersucht werden. Durch die Steuerung von Simulationsläufen in Iterationsschleifen können auch erforderliche Parameteränderungen zur Erreichung bestimmter Zielwerte ermittelt werden.

\section{Einleitung}

Materialflusssysteme kann man im einfachsten Fall als eine Reihenschaltung von Förder- und Bedienelementen verstehen. Ist die Verweilzeit in jedem Element Dirac-verteilt, liegen also konstante Taktzeiten vor, so lässt sich die gesamte Durchlaufzeit durch das System als Summe aller Einzel-Taktzeiten trivial errechnen und der Reziprokwert stellt den Durchsatz D dar.

In der Realität können verschiedenste Einflüsse zu einer Reduzierung dieses Durchsatzes führen:

Störungen können bis zu deren Behebung eine Unterbrechung im Materialfluss bewirken, wobei sie zum Stillstand des gesamten Systems führen können, oder bei Vorhandensein von Puffern nur Teilbereiche betreffen können.

Stochastisch zeitverteilte Störungen können durch die Verfügbarkeit ๆi des Elementes quantitativ erfasst werden. Sind alle Verfügbarkeitswerte der in Reihe geschalteten Elemente bekannt, so kann die Gesamtverfügbarkeit eines aus n Elementen bestehenden Systems nach

$$
\eta_{\text {ges }}=\prod_{i}^{n} \eta_{i}
$$

errechnet werden. Um einen Solldurchsatz $\mathrm{D}_{\text {soll }}$ zu gewährleisten, muss daher das System in der ungestörten Betriebszeit diesen erreichen und daher auf einen höheren technischen Durchsatz $\mathrm{D}_{\text {tech }}$ ausgelegt werden.

$$
D_{\text {tech }}=\frac{D_{\text {soll }}}{\eta_{\text {ges }}}
$$

\section{Erhöhung der Verfügbarkeit durch Redundanz}

Um diesen technischen Durchsatz niedrig zu halten, muss also die Gesamtverfügbarkeit des Systems möglichst hoch sein. Wird diese Gesamtverfügbarkeit durch ein einziges kritisches Element oder durch wenige Elemente sehr negativ beeinflusst und kann man deren Einzelverfügbarkeit nicht mit angemessenem Aufwand erhöhen, so kann man die Verfügbarkeit dieser Elemente durch Redundanz wesentlich erhöhen.

Von kalter Redundanz spricht man, wenn ein parallel geschaltetes Element in Stand-by-Schaltung bei Störung die Aufgabe des gestörten Elementes übernimmt.

Für die Gesamtverfügbarkeit zweier Komponenten in kalter Redundanz ergibt sich nach Birolini [Birolini 1997]:

$$
\eta=\frac{1+\delta}{1+\delta+\delta^{2}} \quad \text { mit } \quad \delta=\frac{M T T R}{M T B F}
$$

MTTR ... Mean Time To Repair (mittlere Stördauer)

MTBF ... Mean Time Between Failures (mittlere störungsfreie Zeit) 
Warme Redundanz liegt dann vor, wenn im einfachsten Fall im störungsfreien Betrieb der Materialfluss auf zwei parallel geschaltete Elemente 1:1 aufgeteilt wird und bei Ausfall eines Elementes der komplette Durchsatz vom in Betrieb verbleibenden Element bewältigt wird. Bei der Ermittlung der erhöhten Verfügbarkeit durch Redundanzschaltung zweier Elemente muss berücksichtigt werden, dass bei warmer Redundanz für die beiden parallel geschalteten Einzelelemente unterschiedliche Einzelverfügbarkeiten gegeben sein werden, je nachdem, ob beide Elemente nur mit je 50\%iger Teillast oder ein Element unter Volllast betrieben wird.

In diesem Fall kann die Gesamtverfügbarkeit der beiden Komponenten in warmer Redundanzschaltung wie folgt berechnet werden [Birolini 1997]:

$$
\eta=\frac{1+2 \delta_{T L}}{1+2 \delta_{T L}+2 \delta_{T L} \delta_{V L}} \quad \text { mit } \quad \begin{aligned}
\delta_{T L} & =\frac{M T T R}{M T B F_{T L}} \\
& \delta_{V L}=\frac{M T T R}{M T B F_{V L}}
\end{aligned}
$$

$$
\begin{gathered}
\text { TL ... Teillast } \\
\text { VL ... Volllast }
\end{gathered}
$$

\section{Puffer zur Reduzierung des technischen Durchsatzes}

Wie bereits erwähnt, beeinflussen Puffer das Systemverhalten entscheidend, wobei deren Begrenztheit in der Größe trotz deren Vorhandenseins zum Gesamtstillstand des Systems führen kann. In diesem Zusammenhang sei auf die VDI-Richtlinie 3649 [VDI-3649 1992] verwiesen. In dieser wird anhand eines Beispiels gezeigt, wie aus einer empirisch ermittelten Häufigkeitsverteilung der Störzeiten direkt abgelesen werden kann, welche Pufferkapazität in Minuten welchen Störzeitanteil abdeckt.

\begin{tabular}{|c|c|c|}
\hline Klasse & $\begin{array}{c}\text { Anzahl der } \\
\text { Störungen }\end{array}$ & $\begin{array}{c}\text { Gesamte Stördauer } \\
\text { in dieser Klasse } \\
(\mathrm{min})\end{array}$ \\
\hline $2-<4$ & 56 & 168 \\
\hline $4-<6$ & 13 & 65 \\
\hline $6-<8$ & 7 & 49 \\
\hline $8-<10$ & 9 & 81 \\
\hline $10-<12$ & 0 & 0 \\
\hline $12-<14$ & 0 & 0 \\
\hline $14-<16$ & 7 & 104 \\
\hline $16-<18$ & 0 & 0 \\
\hline $18-<20$ & 3 & 54 \\
\hline $20-<22$ & 0 & 0 \\
\hline $22-<24$ & 2 & 46 \\
\hline $24-<26$ & 0 & 0 \\
\hline $26-<28$ & 1 & 27 \\
\hline $28-<30$ & 0 & 0 \\
\hline $30-<32$ & 0 & 0 \\
\hline $32-<34$ & 0 & 0 \\
\hline $34-<36$ & 0 & 0 \\
\hline $36-<38$ & 1 & 37 \\
\hline $38-<40$ & 0 & 0 \\
\hline $40-<42$ & 0 & 0 \\
\hline $42-<44$ & 0 & 0 \\
\hline $44-<46$ & 0 & 0 \\
\hline $46-<48$ & 1 & 46 \\
\hline Summe & 100 & 677 \\
\hline & & \\
\hline
\end{tabular}

Tabelle 1: Klassifizierte Daten der Stördauer [VDI-3649 1992]

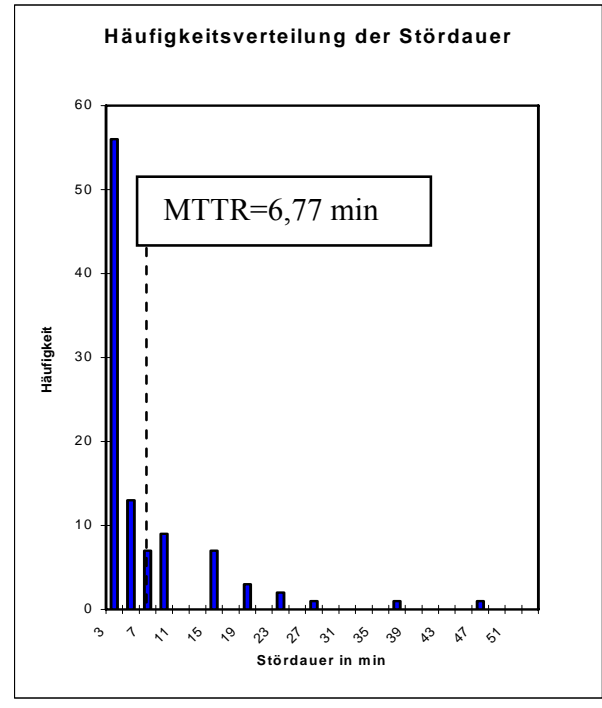

Abbildung 1: Häufigkeitsverteilung der Stördauer [VDI-3649 1992]

Wird z.B. eine Pufferkapazität der Größe MTTR gewählt, so werden alle Störungen kleiner MTTR voll abgedeckt:

$$
168+65=233 \mathrm{~min}
$$

Zusätzlich wird von den Störungen, die größer sind als MTTR, jeweils der Zeitanteil MTTR abgedeckt:

$$
31 \cdot 6,77=210 \mathrm{~min}
$$

Von allen Störungen (677 min) werden also vom Puffer 443 min abgedeckt. Zusammenfassend kann also festgehalten werden, dass bei einer Pufferkapazität x der Größe MTTR und einer typischen Störzeitverteilung nach Abbildung 1, die auch in der Praxis häufig vorzufinden ist, ca. 2/3 aller Störungen durch den Puffer abgedeckt werden. 
In [Decker 2006] wird dieser Zusammenhang für eine sehr typische stetige Verteilungsfunktion von Störzeiten, nämlich der Exponentialverteilung, hergeleitet:

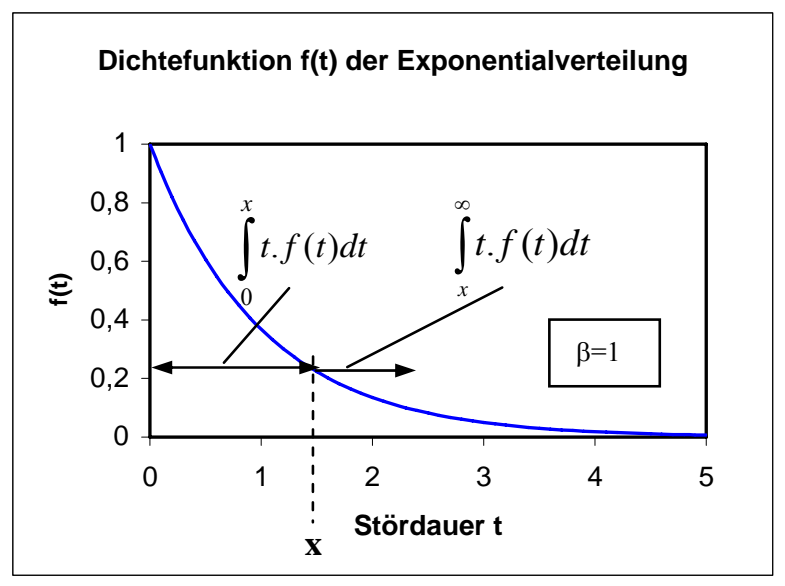

Die Dichtefunktion $\mathrm{f}(\mathrm{t})$ der Exponentialverteilung mit konstantem Parameter $\beta>0$ ist für $\mathrm{t} \geq 0$ folgendermaßen definiert:

$$
\begin{aligned}
& f(t)=\beta . e^{-\beta . t} \\
& \beta=\frac{1}{E(t)}=\frac{1}{M T T R}
\end{aligned}
$$

\section{Abbildung 2: Dichtefunktion f(t) der Exponentialverteilung [Decker 2006]}

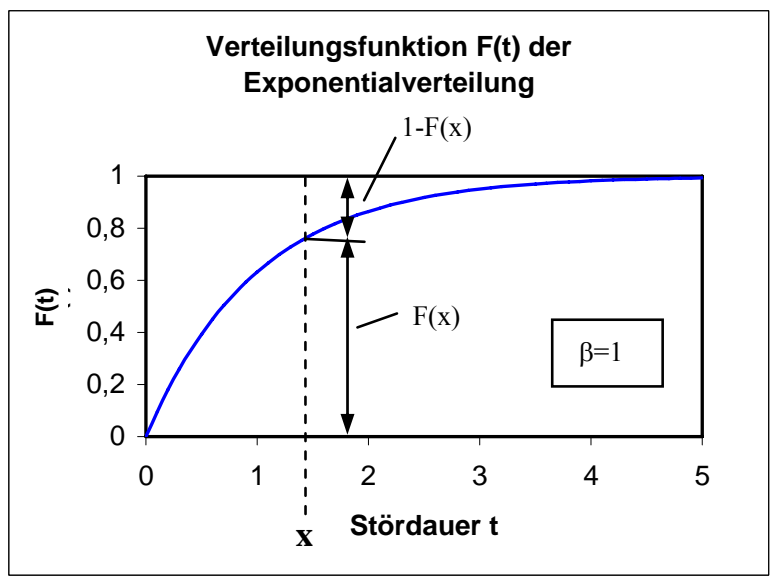

Die Verteilungsfunktion $\mathrm{F}(\mathrm{t})$ der Exponentialverteilung mit konstantem Parameter $\beta>0$ ist für $t \geq 0$ folgendermaßen definiert:

Abbildung 3: Verteilungsfunktion F(t) der Exponentialfunktion [Decker 2006]

Wird jene Zeitspanne (Erwartungswert $\mathrm{E}(\mathrm{x})$ ) berechnet, die im Mittel bei einer bestimmten Pufferkapazität $\mathrm{x}$ in Minuten überbrückt wird, so kann daraus der Überbrückungsanteil f wie folgt berechnet werden:

Der Erwartungswert E(t) der Stördauer kann aus der Dichtefunktion $\mathrm{f}(\mathrm{t})$ folgendermaßen ermittelt werden:

$$
E(t)=\int_{0}^{\infty} t \cdot f(t) d t=\frac{1}{\beta}
$$

Unter Berücksichtigung der beiden Intervalle in Abbildung 2 ergibt sich:

$$
E(t)=\int_{0}^{x} t . f(t) d t+\int_{x}^{\infty} t . f(t) d t
$$

Damit kann die Zeitspanne, die im Mittel bei einer Pufferkapazität x überbrückt wird, mit folgender Überlegung berechnet werden: Alle Störzeiten, die kürzer sind als x werden voll überbrückt. Von den Störungen, die länger dauern als x wird jeweils der Anteil x überbrückt:

$$
E(x)=\int_{0}^{x} t \cdot f(t) d t+\int_{x}^{\infty} x . f(t) d t
$$


Ersetzt man das zweite Integral durch die Verteilungsfunktion F(x), so führt dies zu:

$$
\begin{aligned}
& E(x)=\int_{0}^{x} t \cdot f(t) d t+x(1-F(x)) \\
& E(x)=\beta \cdot \int_{0}^{x} e^{-\beta . t} \cdot t d t+x \cdot e^{-\beta \cdot x}
\end{aligned}
$$

Das Integral kann mittels partieller Integration gelöst werden und führt zu folgendem Ausdruck:

$$
E(x)=-x \cdot e^{-\beta \cdot x}-\frac{1}{\beta} \cdot e^{-\beta \cdot x}+\frac{1}{\beta}+x \cdot e^{-\beta \cdot x}=\frac{1}{\beta} \cdot\left(1-e^{-\beta \cdot x}\right)
$$

Für den Überbrückungsanteil f ergibt sich schließlich:

$$
\begin{aligned}
& f=\frac{E(x)}{E(t)}=\beta \cdot E(x)=1-e^{-\beta \cdot x}=F(x) \\
& f=1-e^{-\beta \cdot x}=F(x)
\end{aligned}
$$

X...Pufferkapazität in Minuten

$\beta$...Reparaturrate (=1/MTTR)

$\mathrm{F}(\mathrm{x})$...Funktionswert für eine Stördauer $\mathrm{x}$

Wird z.B. eine Pufferkapazität $\mathrm{x}$ der Größe MTTR gewählt, also $\mathrm{x}=1 / \beta$, dann werden $63 \%(\mathrm{f}=0,63)$ aller Störungen überbrückt. Dies entspricht etwa dem Wert $2 / 3$ bei der zuvor betrachteten empirischen Verteilungsfunktion gemäß [VDI-3649 1992]. Die in Abbildung 1 dargestellte Häufigkeitsverteilung entspricht ja tatsächlich ungefähr einer Exponentialverteilung.

\section{Analytische Bestimmung des erforderlichen technischen Durchsatzes}

Gemäß [VDI-3649 1992] sowie [Decker 2006] kann der erforderliche technische Durchsatz der Anlagenkomponenten analytisch folgendermaßen bestimmt werden:

Ein Puffer, der zwischen zwei Anlagenteilen angeordnet ist, hat zwei Funktionen. Er nimmt Teile auf, wenn die nachfolgenden Elemente gestört sind und er gibt Teile ab, wenn die vorgeschalteten Elemente stillstehen. Deshalb wird der Puffer für die Berechnung des erforderlichen technischen Durchsatzes einmal dem vorgelagerten Anlagenteil (Anlagenteil I) und einmal dem nachfolgenden Anlagenteil (Anlagenteil II) zugeordnet.

Pufferzuordnung zu Anlagenteil I (vorgeschalteter Puffer für Anlagenteil II, Ausgangslage: Puffer voll)

Kommt es in Anlagenteil I zu einer störungsbedingten Unterbrechung des Materialflusses, so können die Elemente des Anlagenteiles II noch solange arbeiten, bis der Puffer leer ist. Der Puffer bewirkt somit eine Erhöhung der Verfügbarkeit des Anlagenteiles I. 


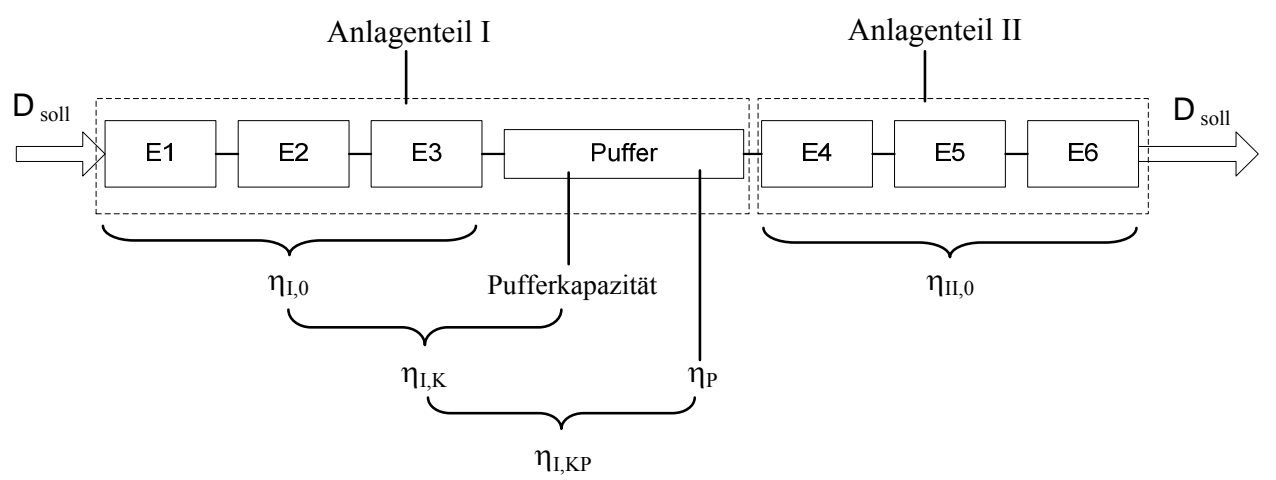

Abbildung 4: Reihenschaltung mit Puffer (Zuordnung zu Anlagenteil I)

Die Gesamtverfügbarkeiten der Anlagenteile I und II ohne Puffer betragen:

$$
\begin{array}{lr}
\eta_{I, 0}=\prod_{i=1}^{p} \eta_{i} & \eta_{I I, 0}=\prod_{i=p+1}^{o} \eta_{i} \\
\begin{array}{l}
\text { p...Anzahl der Elemente } \\
\text { vor dem Puffer }
\end{array} & \text { o...Anzahl aller Elemente }
\end{array}
$$

Die Verfügbarkeit des Anlagenteiles I ohne Puffer kann auch folgendermaßen ausgedrückt werden:

$$
\begin{aligned}
& \eta_{I, 0}=\frac{T_{B}-T_{T}}{T_{B}}=1-\frac{T_{T}}{T_{B}} \\
& \mathrm{~T}_{\mathrm{B}} \ldots \text { Belegungszeit } \\
& \mathrm{T}_{\mathrm{T} \ldots \text { technische Ausfallzeit }}
\end{aligned}
$$

Durch den Puffer wird ein Anteil f der Störzeiten im Anlagenteil I abgedeckt. Um diese Störzeit ist daher $\mathrm{T}_{\mathrm{T}} \mathrm{zu}$ reduzieren:

$$
\eta_{I, K}=\frac{T_{B}-\left(T_{T}-f . T_{T}\right)}{T_{B}}=\frac{T_{B}-T_{T}}{T_{B}}+f \cdot \frac{T_{T}}{T_{B}}
$$

Für die Verfügbarkeit des Anlagenteiles I mit Berücksichtigung der Pufferkapazität ergibt sich somit:

$$
\eta_{I, K}=\eta_{I, 0}+f\left(1-\eta_{I, 0}\right)
$$

f...Anteil der Störzeit, die bei einer bestimmten Pufferkapazität überbrückt werden kann

Soll auch die Verfügbarkeit des Puffers berücksichtigt werden, muss dieser Wert noch mit $\eta_{P}$ multipliziert werden:

$$
\eta_{I, K P}=\eta_{I, K} \cdot \eta_{P}
$$

Der erforderliche technische Durchsatz beträgt dann:

$$
D_{I I, \text { tech }}=\frac{D_{\text {soll }}}{\eta_{I, K P} \cdot \eta_{I I, 0}}
$$

Die Pufferkapazität kann nach folgender Formel berechnet werden:

$$
K_{I I}=x \cdot D_{I I \text { trech }}=x \cdot \frac{D_{\text {soll }}}{\left[\eta_{I, 0}+f\left(1-\eta_{I, 0}\right)\right] \eta_{P} \cdot \eta_{I I, 0}}
$$

x...Pufferkapazität in Zeiteinheiten 
Pufferzuordnung zu Anlagenteil II (nachgeschalteter Puffer für Anlagenteil I, Ausgangslage: Puffer leer)

Kommt es in Anlagenteil II zu einer störungsbedingten Unterbrechung des Materialflusses, so können die Elemente des Anlagenteiles I noch solange arbeiten, bis der Puffer voll ist. Der Puffer bewirkt somit eine Erhöhung der Verfügbarkeit des Anlagenteiles II.

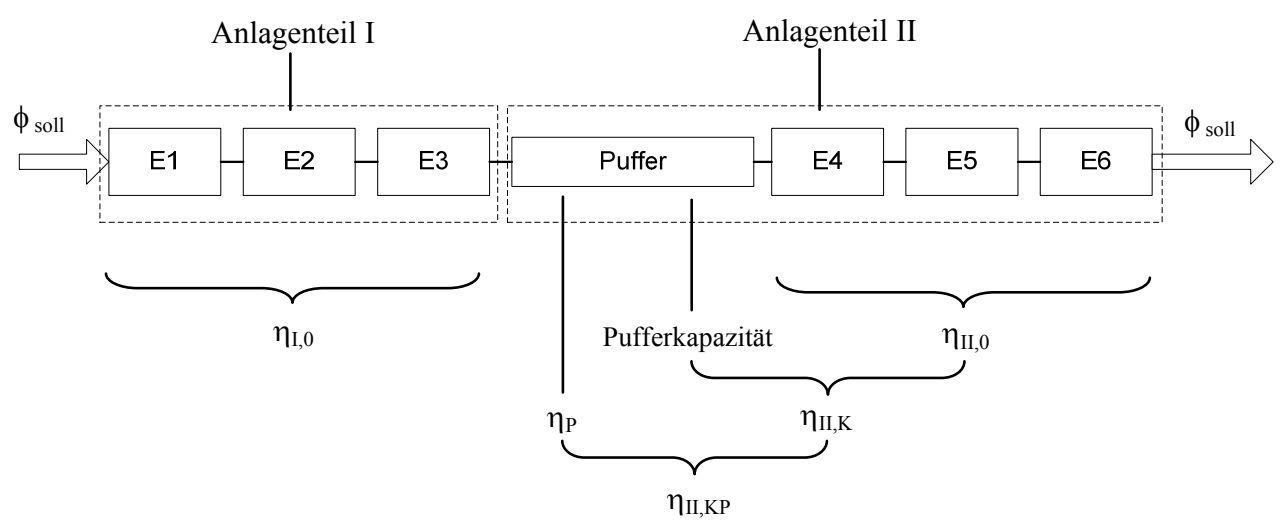

Abbildung 5: Reihenschaltung mit Puffer (Zuordnung zu Anlagenteil II)

In diesem Fall ergibt sich für die Verfügbarkeit des Anlagenteiles II mit Berücksichtigung der Pufferkapazität:

$$
\eta_{I I, K}=\eta_{I I, 0}+f\left(1-\eta_{I I, 0}\right)
$$

Mit Berücksichtigung der Pufferverfügbarkeit erhält man:

$$
\eta_{I I, K P}=\eta_{I I, K} \cdot \eta_{P}
$$

Der erforderliche technische Durchsatz beträgt dann:

$$
D_{I, \text { tech }}=\frac{D_{\text {soll }}}{\eta_{I, 0} \cdot \eta_{I I, K P}}
$$

Die Pufferkapazität kann nach folgender Formel berechnet werden:

$$
K_{I}=x \cdot D_{I, \text { tech }}=x \cdot \frac{D_{\text {soll }}}{\left[\eta_{I I, 0}+f\left(1-\eta_{I I, 0}\right)\right] \cdot \eta_{P} \cdot \eta_{I, 0}}
$$

Soll ein doppeltwirkender Puffer installiert werden, so ist folgende Pufferkapazität erforderlich:

$$
K_{g e s}=K_{I}+K_{I I}
$$

Im Ausgangszustand muss $\mathrm{K}_{\mathrm{I}}$ leer und $\mathrm{K}_{\mathrm{II}}$ voll sein.

Wird ein Puffer mit ausreichender Kapazität installiert, sodass alle Störungen abgedeckt werden können, führt dies zu einer vollständigen Entkopplung der beiden Anlagenteile. Der erforderliche technische Durchsatz beträgt somit bei $\mathrm{f}=1$ :

$$
D_{I, \text { tech }}=\frac{D_{\text {soll }}}{\eta_{I, 0} \cdot \eta_{P}} \quad D_{I I, \text { tech }}=\frac{D_{\text {soll }}}{\eta_{I I, 0} \cdot \eta_{P}}
$$

Puffer beeinflussen das Systemverhalten aber nicht nur insofern positiv, als damit Störzeiten überbrückt werden können, sondern dienen auch als Ausgleichselemente, wenn die Transport- und Bedienzeiten schwanken können, die sogenannten Zwischenankunftszeiten von Element zu Element also unterschiedlich sein können, sodass sich vor einem Element Warteschlangen bilden können. Selbstverständlich muss der Erwartungswert der Zwischenankunftszeiten des Elementes i größer/gleich dem Erwartungswert am Folgeelement (i+1) sein, damit die Warteschlage bei Fortdauer des Betriebes nicht gegen unendlich strebt. Aber selbst bei Erfüllung dieser Bedingung wird die Begrenztheit des Puffers als Ausgleichselement zu Unterbrechungen im Materialfluss führen. 


\section{Untersuchung des Systemverhaltens durch Simulation}

Wollte man alle diese Einflüsse unter stochastischer Verteilung der Zwischenankunftszeiten und Störzeiten in einer analytischen Rechnung berücksichtigen, so stößt man schon bei relativ einfachen mehrgliedrigen Systemen an Grenzen. Daher lassen sich Untersuchungen zielführend nur mit diskreter Simulation durchführen.

In [Decker 2006] wurden mit dem Simulationspaket ARENA, welches auf der Simulationssprache SIMAN basiert, spezielle ein Materialflusssystem charakterisierende Module entwickelt, mit denen die Modellierung eines Systems relativ einfach erfolgen kann. Dabei können nicht nur Störungen aller Materialflusskomponenten und Puffer mit beschränkten Kapazitäten, sondern auch redundant ausgeführte Transportmittel, bzw. Bedienstationen berücksichtigt werden. Das komplette Materialflusssystem wird durch eine Vielzahl von Animationselementen am Bildschirm visualisiert und eine umfangreiche Onlinestatistik, wie z.B. Auslastungsgrade, Blockierzeiten, Ausfallzeiten, technische Verfügbarkeiten, Warteschlangenlängen und Durchlaufzeiten, ausgegeben.

Bei einer Untersuchung mit Hilfe der Simulation können grundsätzlich nur auf Grund von vorgegebenen Daten, welche die Systemeigenschaften beschreiben, Ergebnisse ermittelt werden; in diesem Fall also insbesondere ein sich daraus im eingeschwungenen Zustand des Systems ergebender Durchsatz. D.h., es kann nicht umgekehrt ein gewünschter Durchsatz vorgegeben werden, um daraus erforderliche Systemparameter zu errechnen.

\section{Iterative Simulationsläufe zur Erzielung bestimmter Ziel- werte}

In der SIMAN/ARENA-Software ist aber eine als Visual Basic for Applications (VBA) bezeichnet Makrosprache eingebunden, die es erlaubt, Simulationsexperimente im Sinne einer iterativen Annäherung an ein gewünschtes Ergebnis zu steuern. Damit ist es möglich, einen sinnvoll erscheinenden Kennwert des Materialflusssystems auszuwählen und iterativ gesteuert so zu verändern, dass ein Zielwert für den Durchsatz erreicht wird. Dadurch ist es möglich, mit geschickt ausgewählten Kennwerten in relativ wenigen Versuchsläufen eine Optimierung des Gesamtsystems vorzunehmen.

In Abbildung 6 ist schematisch dargestellt, wie die drei Softwaretools ARENA, VBA und MS EXCEL während der Simulationsläufe interagieren.

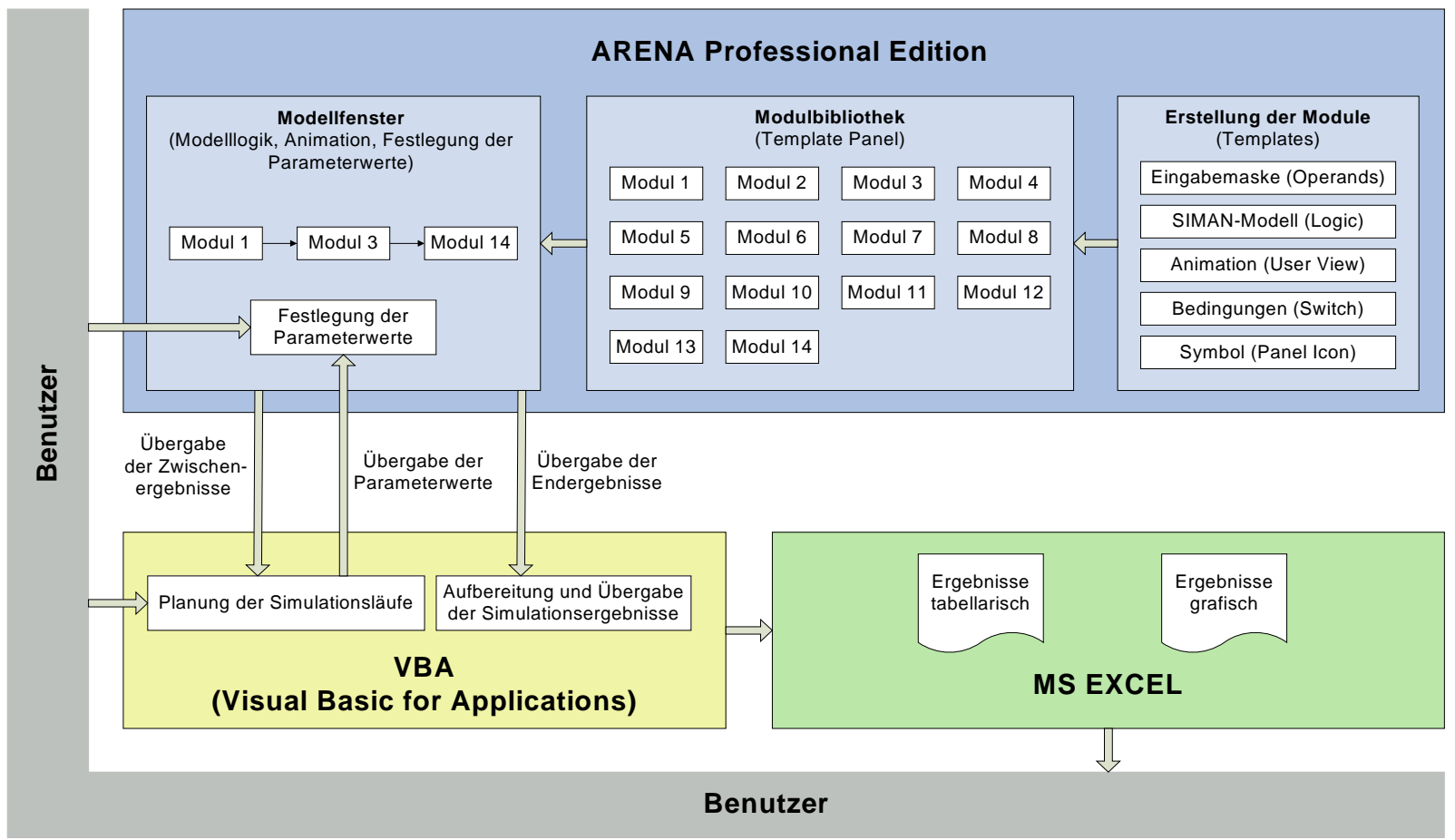

Abbildung 6: Softwarestruktur 
Der Benutzer wählt den entsprechenden Modul aus der Bibliothek aus und platziert diesen auf dem ARENAModellfenster. In übersichtlich gestalteten Dialogboxen können dann alle relevanten Parameterwerte eingegeben werden. Wenn das Simulationsmodell vollständig erstellt wurde, können in einem VBA-Formular die Parameter für die Iterationsroutine eingegeben werden. Dann wird der Simulationslauf gestartet. Die Iterationsroutine wird nun solange durchlaufen, bis der Wert der Ergebnisgröße innerhalb des zuvor definierten Bereiches liegt. Die Ergebnisse werden dann aufbereitet und an MS EXCEL übergeben. Dort können die Simulationsergebnisse tabellarisch oder grafisch dargestellt werden.

Die Bibliothek mit den 14 Modulen ist in Abbildung 7 dargestellt.

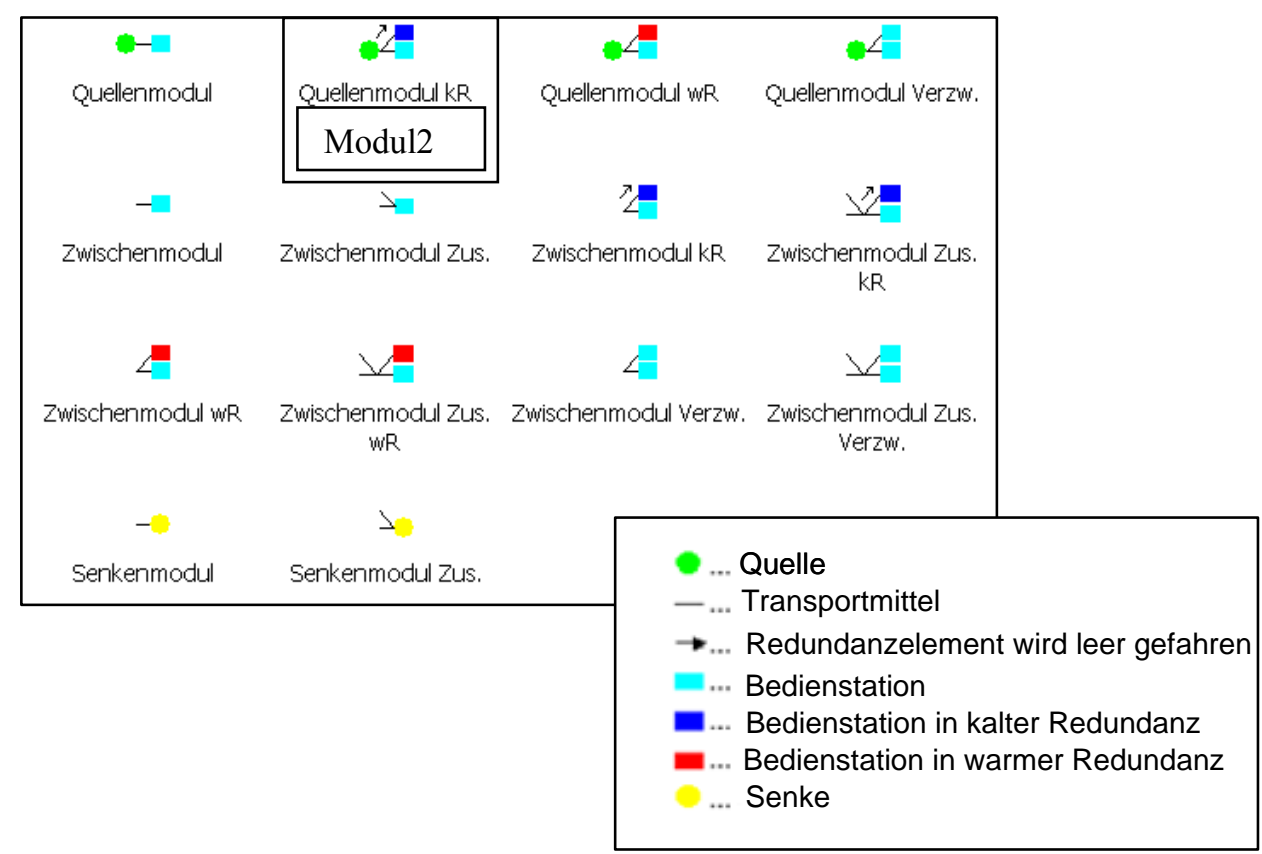

Abbildung 7: Modul-Bibliothek

Exemplarisch wird in Abbildung 8 das Modellkonzept für Modul2 (Quellenmodul mit kalter Redundanzschaltung) beschrieben sowie die Dialogboxen mit den einzugebenden Parametern in Abbildung 9 dargestellt. Schließlich werden noch die am Bildschirm dargestellten Animationselemente in Abbildung 10 veranschaulicht.

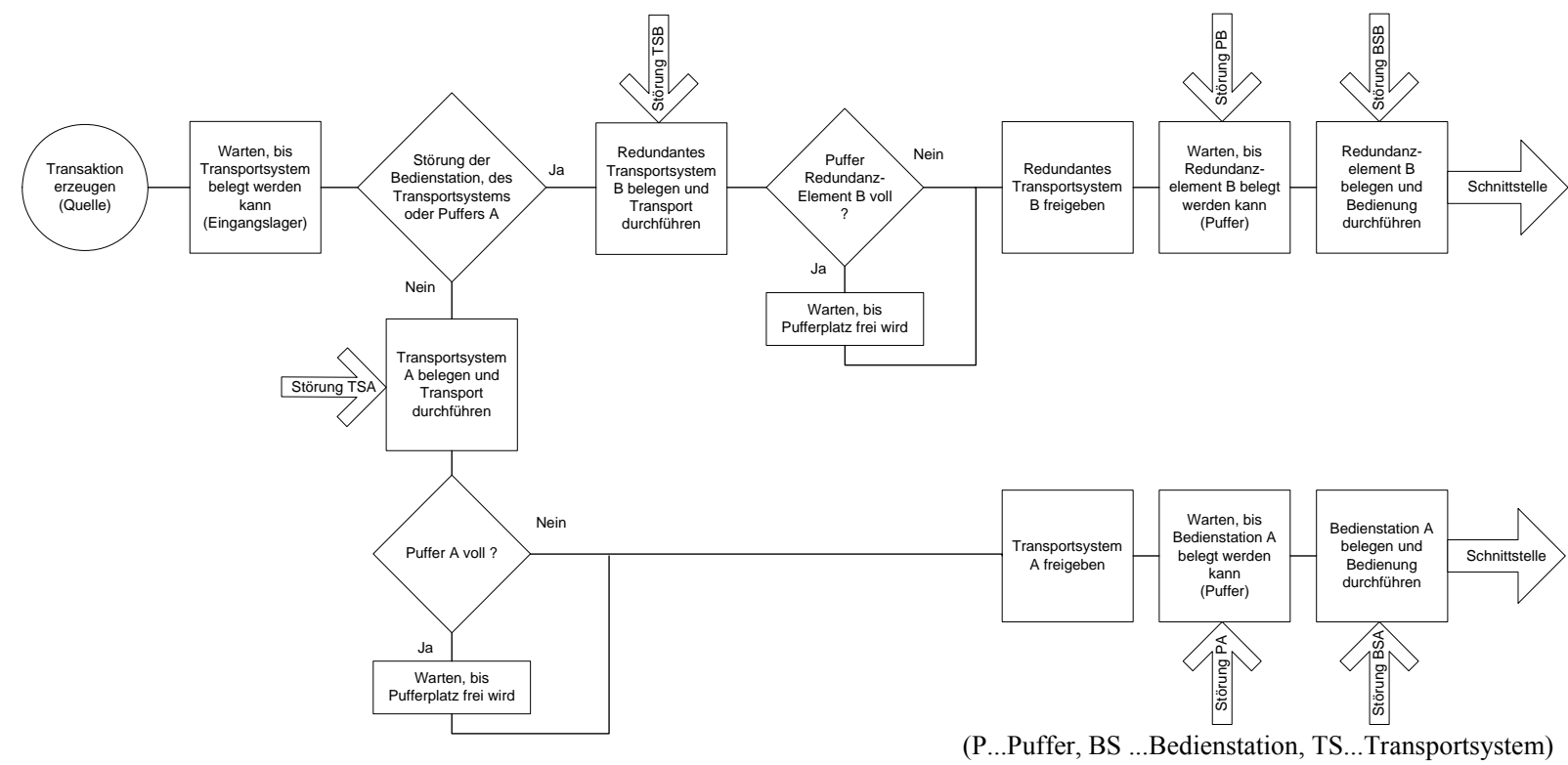

Abbildung 8: Modellkonzept für Modul2 (Quellenmodul mit kalter Redundanzschaltung) 


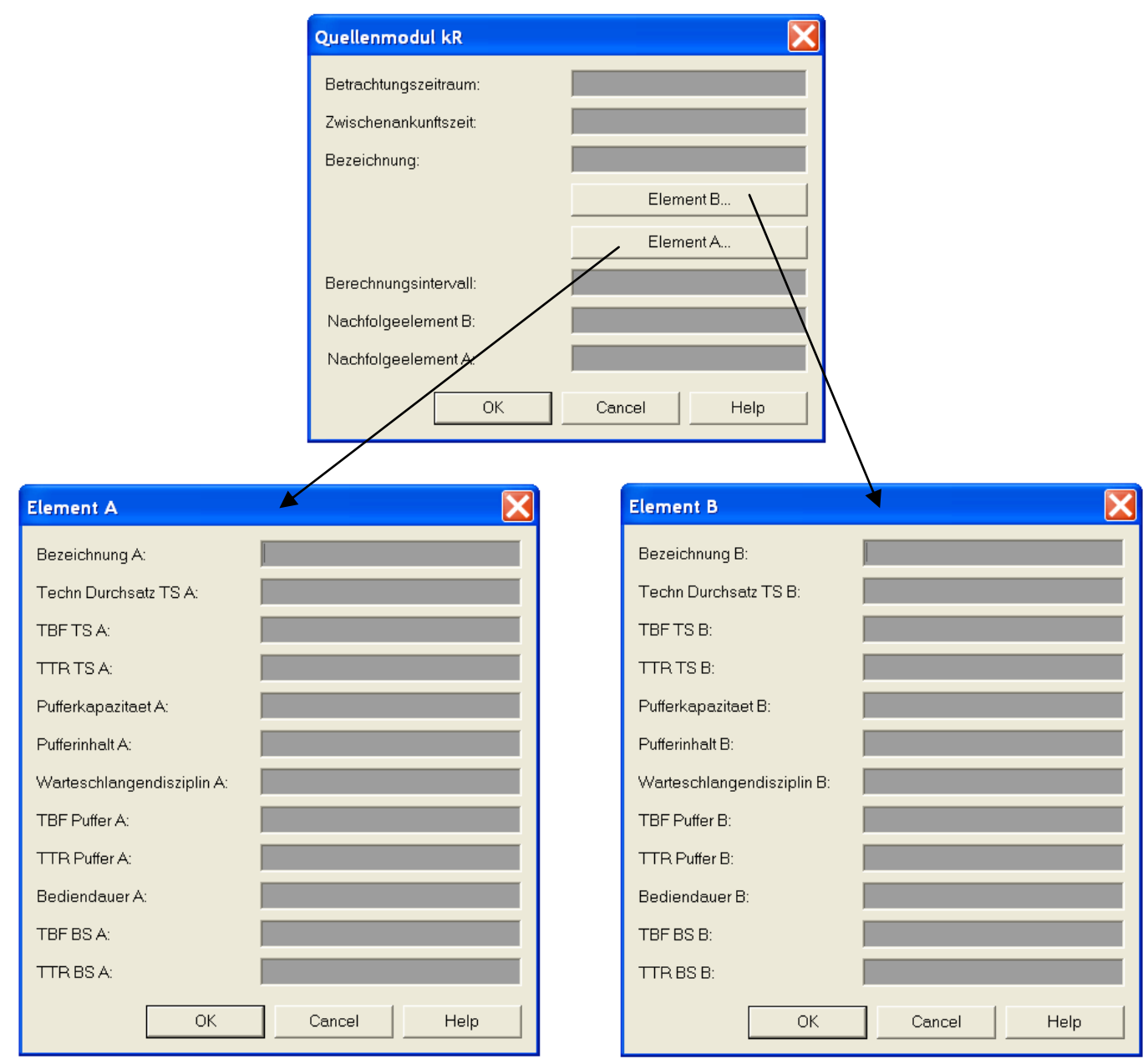

Abbildung 9: Dialogboxen für Modul2 (Quellenmodul mit kalter Redundanzschaltung)

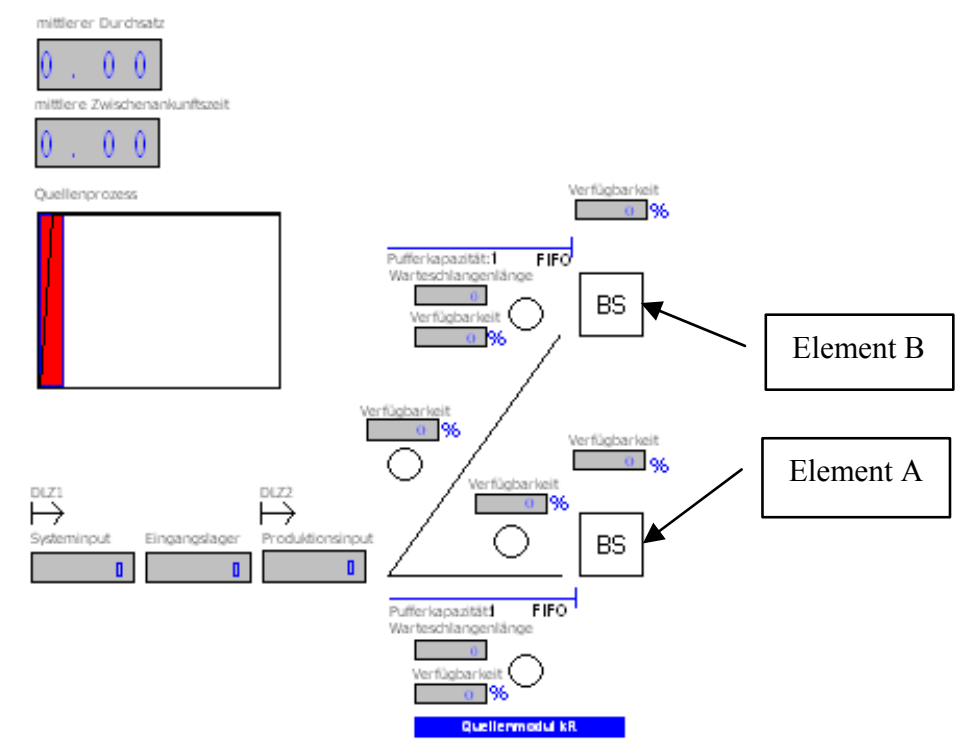

Abbildung 10: Animationselemente für Modul2 (Quellenmodul mit kalter Redundanzschaltung) 
Für die Bestimmung des erforderlichen technischen Durchsatzes von Materialflusselementen mittels Simulation, bei Vorgabe eines gewünschten Anlagendurchsatzes, kann z.B. folgende Vorgangsweise gewählt werden:

\section{1) Basissystem festlegen:}

Für alle relevanten Systemkomponenten (Fördersysteme, Bedienstationen, Puffer) muss das jeweilige Störverhalten (Verteilungsfunktion) und gegebenenfalls auch die Art der Redundanzschaltung bekannt sein. Weiters müssen für alle Puffer entsprechende Pufferkapazitäten angenommen werden.

\section{2) Grenzwerte für die Transport- und Bedienzeiten mittels einfacher Berechnungsverfahren bestimmen:}

Für das Basissystem werden Grenzwerte $\left(\mathrm{P}_{\min }, \mathrm{P}_{\max }\right.$ in Abbildung 11) für die Transport- und Bedienzeiten ermittelt (bei stochastischen Prozessen für die entsprechenden Mittelwerte). Dies kann z.B. dadurch erfolgen, dass die Berechnung einmal ohne Berücksichtigung von Puffern und einmal unter der Annahme unbegrenzter Puffer durchgeführt wird.

\section{3) Mittels iterativer Parametervariation Bedien- und Transportzeiten zur Erreichung des gewünschten Durchsatzes ermitteln:}

Unter Berücksichtigung der unter Punkt 2) ermittelten Grenzwerte werden alle Bedien- und Transportzeiten variiert. Die Iterationsroutine läuft dabei vollkommen automatisiert ab. Das Prinzip der iterativen Parametervariation soll in Abbildung 11 veranschaulicht werden.

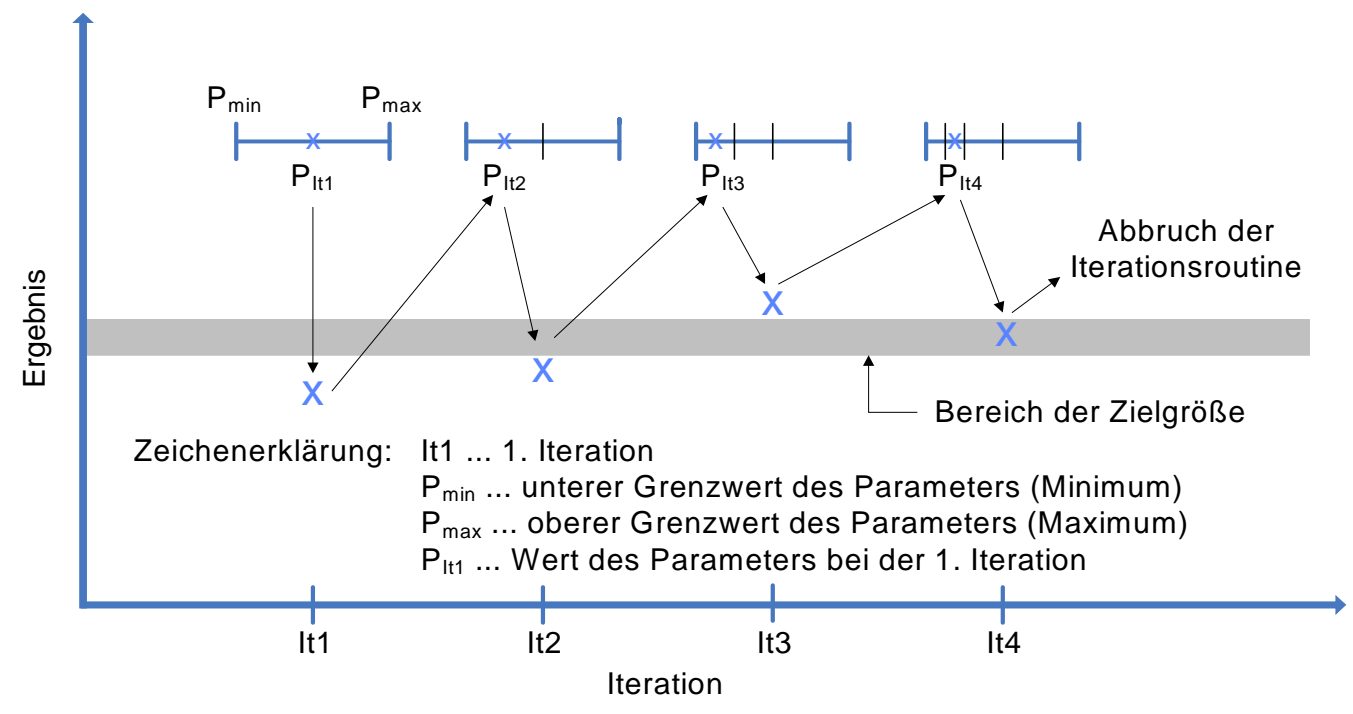

Abbildung 11: Prinzip der iterativen Parametervariation

Die 1. Iteration startet mit dem Wert PIt1 $=(\operatorname{Pmax}+\mathrm{Pmin}) / 2$ (P: Bedien- oder Transportdauer). Dann wird die Simulation durchlaufen und abhängig vom Ergebnis (Anlagendurchsatz) wird der neue Wert des Parameters PIt2 wiederum durch Halbieren des entsprechenden Intervalls gewählt. Dieser Vorgang wird solange wiederholt, bis der geforderte Solldurchsatz erreicht ist. Dieses Verfahren ermöglicht es, bei überlegt gewählten Anfangswerten nach nur wenigen Simulationsläufen das gewünschte Resultat zu erhalten.

\section{4) Optimierung der Systemparameter}

Bei jenen Materialflusskomponenten, die sehr viele Leerzeiten aufweisen, können die Transport bzw. Bedienzeiten, trotz Wahrung des gewünschten Anlagendurchsatzes, erhöht werden. Das Ausmaß der Erhöhung kann durch Simulationsläufe ermittelt werden.

\section{5) Falls erforderlich, Systemkomponenten (Simulationsmodule) austauschen und Ablauf wiederholen}

Können die ermittelten Transport- und Bedienzeiten in der realen Anlage nicht erreicht werden, müssen Änderungen am System vorgenommen werden. Elemente mit besonders schlechter Verfügbarkeit können z.B. durch andere oder weitere Redundanzschaltungen ersetzt werden. Man könnte die Iterationen auch mit anderen Pufferkapazitäten durchführen. 


\section{Beispiel [Decker 2006]}

Es wurde ein Beispiel gewählt, das auch analytisch gelöst werden kann. Dadurch ist es möglich, eine Validation des Simulationsmodells durchzuführen.

Ein Produktionssystem sei mit einer Montagestation starr verknüpft. Das Gesamtsystem setzt sich aus 9 Elementen zusammen:

- 3 Fördersysteme (FS1, FS2, FS3)

- 3 Puffer (P1, P2, P3)

- 2 Produktionssysteme in kalter Redundanzschaltung (PS1, PS2)

- 1 Montagestation (MS)

Anlagenteil I

Techn. Durchsatz $D_{\text {I tech }}$
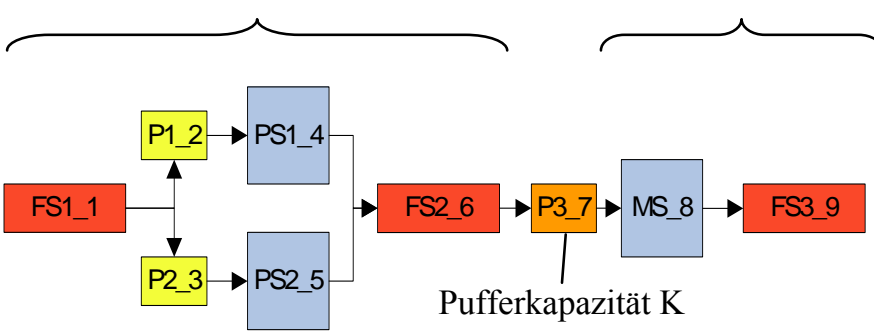

FS1, FS2, FS3, PS1, PS2, MS ... DETERMINISTISCH

FS...Fördersystem / P...Puffer / PS...Produktionssystem / MS...Montagesystem
Anlagenteil II Techn. Durchsatz D II_tech

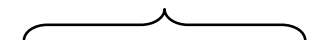

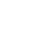
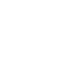
Exemplarisch wird die Berechnung der erforderlichen technischen Durchsätze in den Anlagenteilen I und II für jene Pufferkapazität angeführt, bei der ca. 2/3 aller Störungen überbrückt werden können (d.h. die Pufferkapazität soll so groß sein, dass eine mittlere Stördauer von 30 Minuten überbrückt werden kann, also beträgt $\mathrm{x}=30$ Minuten und $\mathrm{f}=0,63$ ):

Für die Gesamtverfügbarkeit der beiden Produktionssysteme in kalter Redundanzschaltung ergibt sich:

$$
\begin{aligned}
& \delta=\frac{M T T R}{M T B F}=\frac{20}{180}=0,111 \\
& \eta_{45}=\frac{1+\delta}{1+\delta+\delta^{2}}=0,989
\end{aligned}
$$

Die Gesamtverfügbarkeit der Anlagenteile I und II betragen somit:

$$
\begin{aligned}
& \eta_{I, 0}=0,98 \cdot 0,989 \cdot 0,98=0,949 \\
& \eta_{I I, 0}=0,94 \cdot 0,95=0,893
\end{aligned}
$$

Mit Berücksichtigung der Überbrückungswirkung des Puffers P3 (f=0,63) ergeben sich folgende Verfügbarkeiten:

$$
\begin{aligned}
& \eta_{I, K}=\eta_{I, 0}+f\left(1-\eta_{I, 0}\right)=0,981 \\
& \eta_{I I, K}=\eta_{I I, 0}+f\left(1-\eta_{I I, 0}\right)=0,960
\end{aligned}
$$

Da auch der Puffer selbst Störungen unterliegt, müssen diese Werte noch mit der Verfügbarkeit des Puffers P3 multipliziert werden:

$$
\begin{aligned}
& \eta_{I, K P}=\eta_{I, K} \cdot \eta_{P 3}=0,952 \\
& \eta_{I I, K P}=\eta_{I I, K} \cdot \eta_{P 3}=0,931
\end{aligned}
$$

Die erforderlichen technischen Durchsätze in den Anlagenteilen I und II bei einem Solldurchsatz von 100 Stk/h betragen somit:

$$
\begin{aligned}
& D_{I, \text { tech }}=\frac{D_{\text {soll }}}{\eta_{I, 0} \cdot \eta_{I I, K P}}=113,2 \mathrm{Stk} / \mathrm{h} \\
& D_{I I, \text { tech }}=\frac{D_{\text {soll }}}{\eta_{I, K P} \cdot \eta_{I I, 0}}=117,6 \mathrm{Stk} / \mathrm{h}
\end{aligned}
$$

Für die Pufferkapazität K ergibt sich schließlich:

$$
K=K_{I}+K_{I I}=x \cdot D_{I, \text { tech }}+x \cdot D_{I I, \text { tech }}=57+59=116 \text { Stk }
$$

Tabelle 3 zeigt eine Gegenüberstellung der analytischen Ergebnisse und der Simulationsergebnisse unter Anwendung der iterativen Parametervariation bei einer Pufferkapazität von 116 Stück.

Tabelle 3: Gegenüberstellung der Ergebnisse

\begin{tabular}{|l|c|c|}
\hline & Berechnungsverfahren & Simulation \\
\hline $\mathrm{D}_{\mathrm{I}, \text { tech }}$ & $113,2 \mathrm{Stk} / \mathrm{h}$ & $112,6 \mathrm{Stk} / \mathrm{h}$ \\
\hline $\mathrm{D}_{\text {II,tech }}$ & $117,6 \mathrm{Stk} / \mathrm{h}$ & $116,2 \mathrm{Stk} / \mathrm{h}$ \\
\hline
\end{tabular}


Die Berechnungen wurden für verschiedene Pufferkapazitäten x in Minuten durchgeführt. Die sich daraus ergebenden Pufferkapazitäten in Stück sind in der nachfolgenden Tabelle zusammengefasst.

Tabelle 4: Pufferkapazitäten von Puffer P3

\begin{tabular}{|l|l|l|l|l|l|l|}
\hline Pufferkapazität x in min & 10 & 20 & 30 & 40 & 50 & 60 \\
\hline Pufferkapazität K in Stk & 40 & 78 & 116 & 153 & 190 & 227 \\
\hline
\end{tabular}

Exemplarisch werden in Abbildungen 13 die Ergebnisse für die erforderlichen technischen Durchsätze im Anlagenteil II, also in der Montagestation, bei den verschiedenen Pufferkapazitäten nach Tabelle 4 gegenübergestellt.

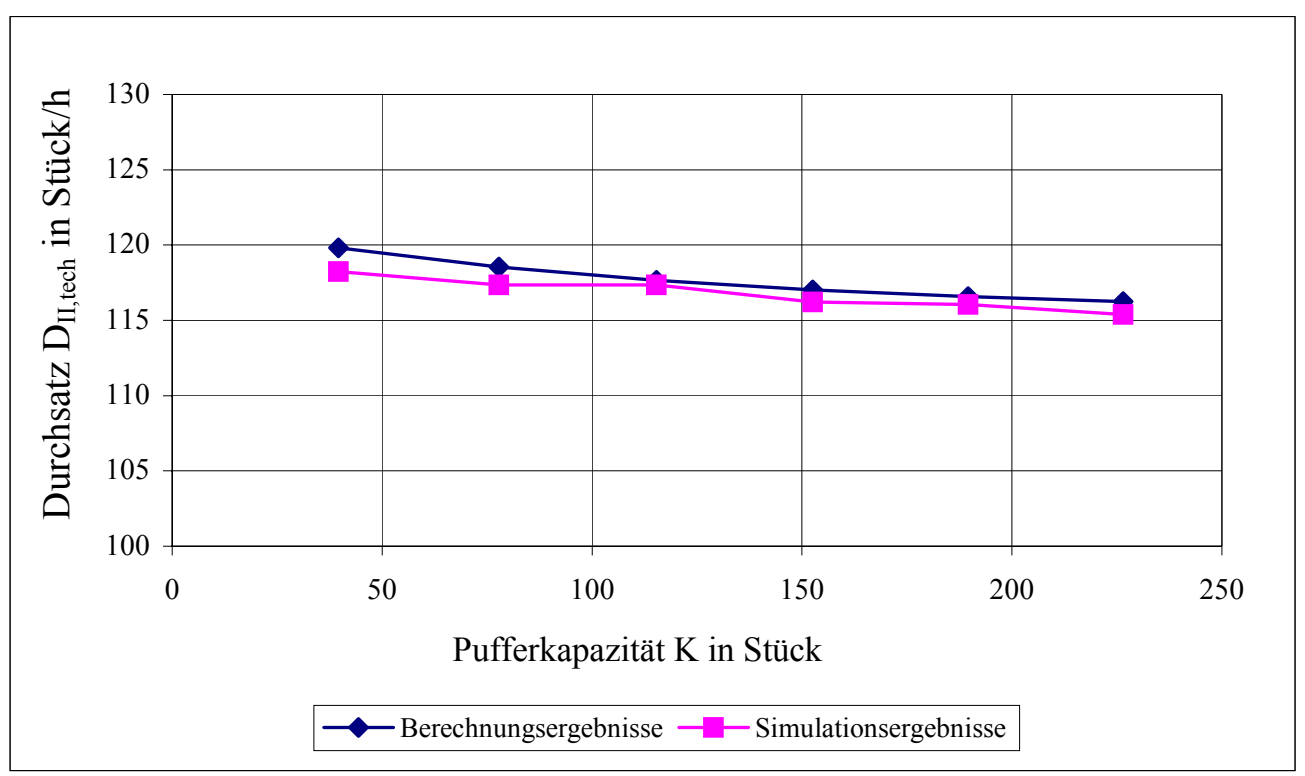

Abbildung 13: Gegenüberstellung der Ergebnisse

\section{Literatur}

[Birolini 1997]

[VDI-3649 1992]

[Decker 2006]
Birolini, Alessandro: Zuverlässigkeit von Geräten und Systemen. 4. Aufl. Berlin [u.a.]: Springer-Verlag, 1997

VDI 3649: Anwendung der Verfügbarkeitsrechnung für Förder- und Lagersysteme. Berlin: Beuth Verlag, 1992

Decker, Klaus: Bestimmung des erforderlichen technischen Durchsatzes von Materialflusselementen mittels diskreter Simulation. Dissertation TU-Wien, 2006 Volltext : http://www.ub.tuwien.ac.at/diss/AC05032786.pdf (Datum des letzten Zugriffs 13.05.2008) 